ISSN 1600-5368

\section{Crystal structure of bromidobis(naph- thalen-1-yl)antimony(III)}

\section{Omar bin Shawkataly, ${ }^{\mathrm{a} *} \neq$ Hafiz Malik Hussien \\ Abdelnasir ${ }^{\mathrm{a}}$ and Mohd Mustaqim Rosli ${ }^{\mathrm{b}}$}

${ }^{a}$ Chemical Sciences Programme, School of Distance Education, Universiti Sains Malaysia, 11800 USM, Penang, Malaysia, and ${ }^{\mathbf{b} X-r a y ~ C r y s t a l l o g r a p h y ~ U n i t, ~ S c h o o l ~ o f ~}$ Physics, Universiti Sains Malaysia, 11800 USM, Penang, Malaysia. *Correspondence e-mail: omarsa@usm.my

Received 30 August 2014; accepted 5 September 2014

Edited by H. Ishida, Okayama University, Japan

In the title compound, $\left[\mathrm{SbBr}\left(\mathrm{C}_{10} \mathrm{H}_{7}\right)_{2}\right]$, the $\mathrm{Sb}^{\mathrm{III}}$ atom has a distorted trigonal-pyramidal coordination geometry and the planes of the two naphthalene ring systems make a dihedral angle of $80.26(18)^{\circ}$. An intramolecular $\mathrm{C}-\mathrm{H} \cdots \mathrm{Br}$ hydrogen bond forms an $S(5)$ ring motif. In the crystal, weak $\mathrm{C}-\mathrm{H} \cdots \mathrm{Br}$ interactions link the molecules into helical chains along the $b$ axis direction.

Keywords: crystal structure; organoantimony(III) compounds; stibine; hydrogen bonding.

CCDC reference: 1023098

\section{Related literature}

For general background to organoantimony(III) compounds and related structures of haloorganoantimony(III) compounds, see: Breunig et al. (2008); Millington \& Sowerby (1994).

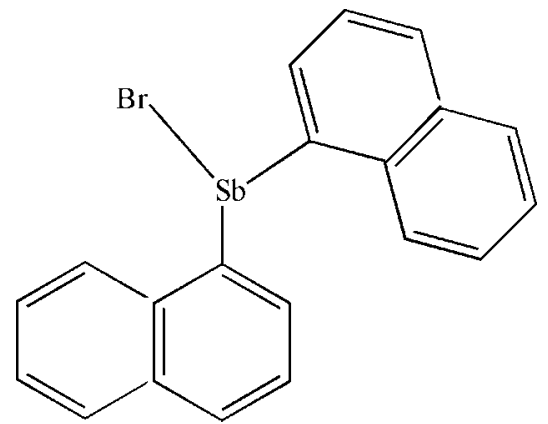

\section{Experimental}

\subsection{Crystal data}

$\left[\operatorname{SbBr}\left(\mathrm{C}_{10} \mathrm{H}_{7}\right)_{2}\right]$

$M_{r}=455.97$

Monoclinic, $P 2_{1} / c$

$a=12.7371(3) \AA$

$b=10.9189(3) \AA$

$c=11.6300(3) \AA$

$\beta=92.661$ (1)

$$
\begin{aligned}
& V=1615.70(7) \AA^{3} \\
& Z=4 \\
& \text { Mo } K \alpha \text { radiation } \\
& \mu=4.17 \mathrm{~mm}^{-1} \\
& T=100 \mathrm{~K} \\
& 0.56 \times 0.33 \times 0.14 \mathrm{~mm}
\end{aligned}
$$

\subsection{Data collection}

Bruker SMART APEXII CCD area-detector diffractometer

Absorption correction: multi-scan (SADABS; Bruker, 2009)

$T_{\min }=0.204, T_{\max }=0.597$

20528 measured reflections 4705 independent reflections 3936 reflections with $I>2 \sigma(I)$ $R_{\text {int }}=0.032$

\subsection{Refinement}

$R\left[F^{2}>2 \sigma\left(F^{2}\right)\right]=0.060$

$w R\left(F^{2}\right)=0.198$

$S=1.06$

4705 reflections

199 parameters

$\mathrm{H}$-atom parameters constrained

$\Delta \rho_{\max }=3.68 \mathrm{e} \AA^{-3}$

$\Delta \rho_{\min }=-3.12 \mathrm{e} \AA^{-3}$

Table 1

Hydrogen-bond geometry $\left(\AA,^{\circ}\right)$.

\begin{tabular}{lllll}
\hline$D-\mathrm{H} \cdots A$ & $D-\mathrm{H}$ & $\mathrm{H} \cdots A$ & $D \cdots A$ & $D-\mathrm{H} \cdots A$ \\
\hline $\mathrm{C} 1-\mathrm{H} 1 A \cdots \mathrm{Br} 1$ & 0.95 & 2.71 & $3.408(6)$ & 130 \\
$\mathrm{C} 2-\mathrm{H} 2 A \cdots \mathrm{Br} 1^{\mathrm{i}}$ & 0.95 & 2.96 & $3.698(6)$ & 135 \\
\hline Symmetry code: (i) $-x, y-\frac{1}{2}-z-\frac{1}{2}$ & & &
\end{tabular}

Symmetry code: (i) $-x, y-\frac{1}{2},-z-\frac{1}{2}$.

Data collection: APEX2 (Bruker, 2009); cell refinement: SAINT (Bruker, 2009); data reduction: $S A I N T$; program(s) used to solve structure: SHELXTL (Sheldrick, 2008); program(s) used to refine structure: SHELXTL; molecular graphics: SHELXTL; software used to prepare material for publication: SHELXTL and PLATON (Spek, 2009).

\section{Acknowledgements}

The authors would like to thank the Malaysian Government and Universiti Sains Malaysia (USM) for Research Grant 1001/PJJAUH/811225. HMHA thanks USM for the award of a USM Fellowship.

Supporting information for this paper is available from the IUCr electronic archives (Reference: IS5373).

\section{References}

Breunig, H. J., Lork, E., Moldovan, O. \& Rat, C. I. (2008). J. Organomet. Chem. 693, 2527-2534.

Bruker (2009). APEX2, SAINT and SADABS. Bruker AXS Inc., Madison, Wisconsin, USA.

Millington, P. L. \& Sowerby, D. B. (1994). J. Organomet. Chem. 480, 227-234. Sheldrick, G. M. (2008). Acta Cryst. A64, 112-122.

Spek, A. L. (2009). Acta Cryst. D65, 148-155. 


\section{supporting information}

Acta Cryst. (2014). E70, m351 [doi:10.1107/S1600536814020066]

\section{Crystal structure of bromidobis(naphthalen-1-yl)antimony(III)}

\section{Omar bin Shawkataly, Hafiz Malik Hussien Abdelnasir and Mohd Mustaqim Rosli}

\section{S1. Experimental}

All synthetic reactions were performed under dry, oxygen-free dinitrogen atmosphere using standard Schlenk techniques; THF was dried over sodium and distilled from sodium benzophenone ketyl under nitrogen. Antimony trichloride, 1bromo-naphthaline, and magnesium filing purchased from Sigma Aldrich. The title compound was prepared by adding a solution of antimony trichloride $(0.9124 \mathrm{~g}, 0.0040 \mathrm{~mol})$ in $30 \mathrm{ml}$ THF was added dropwise with stirring to a Grignard mixture of magnesium filings $(0.31 \mathrm{~g}, 0.0129 \mathrm{~mol})$ and 1-bromonaphthaline $(1.72 \mathrm{~g}, 0.0083 \mathrm{~mol})$. The reaction mixture was stirred for $12 \mathrm{~h}$, the solvent was removed in vacuum and the remaining solid was recrystallized from ethanol.

\section{S2. Refinement}

All $\mathrm{H}$ atoms were positioned geometrically and refined using a riding model with with $\mathrm{C}-\mathrm{H}=0.95 \AA$ and $U_{\text {iso }}(\mathrm{H})=$ $1.2 U_{\mathrm{eq}}(\mathrm{C})$.

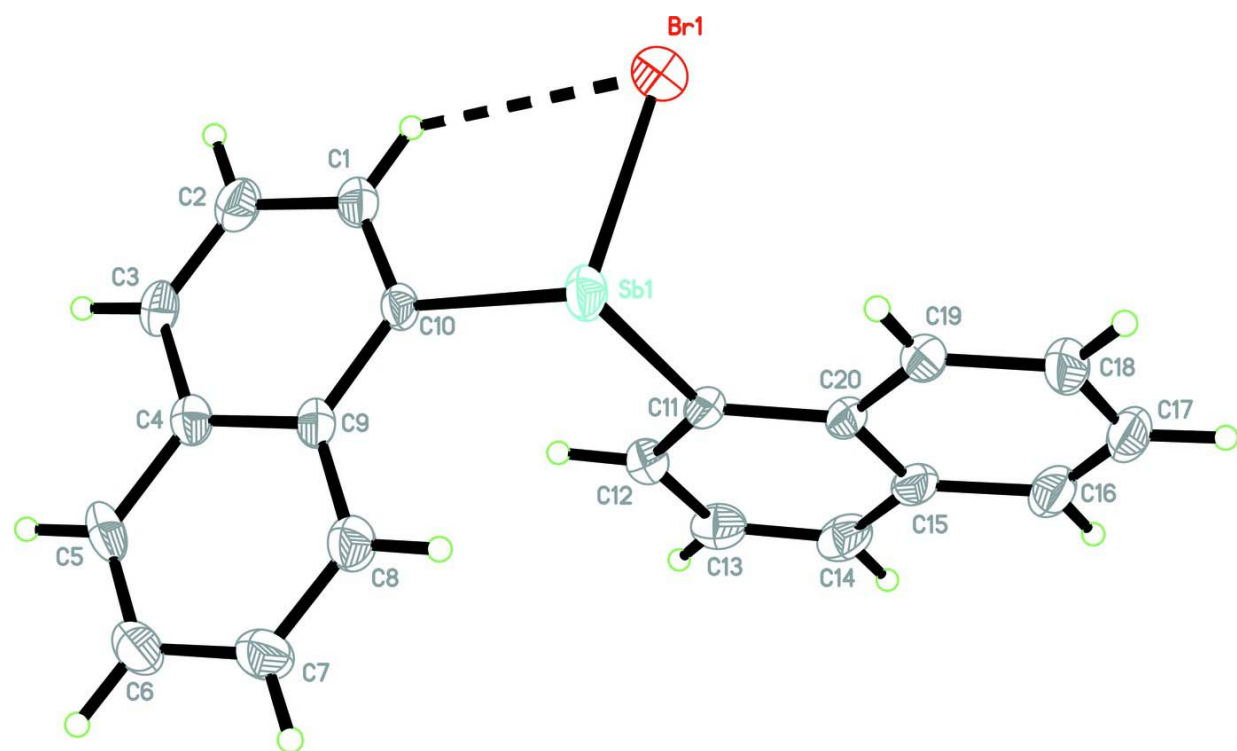

Figure 1

The molecular structure of the title compound, showing 50\% probability displacement ellipsoids. The dashed line indicates the $\mathrm{C}-\mathrm{H} \cdots \mathrm{Br}$ hydrogen bond. 


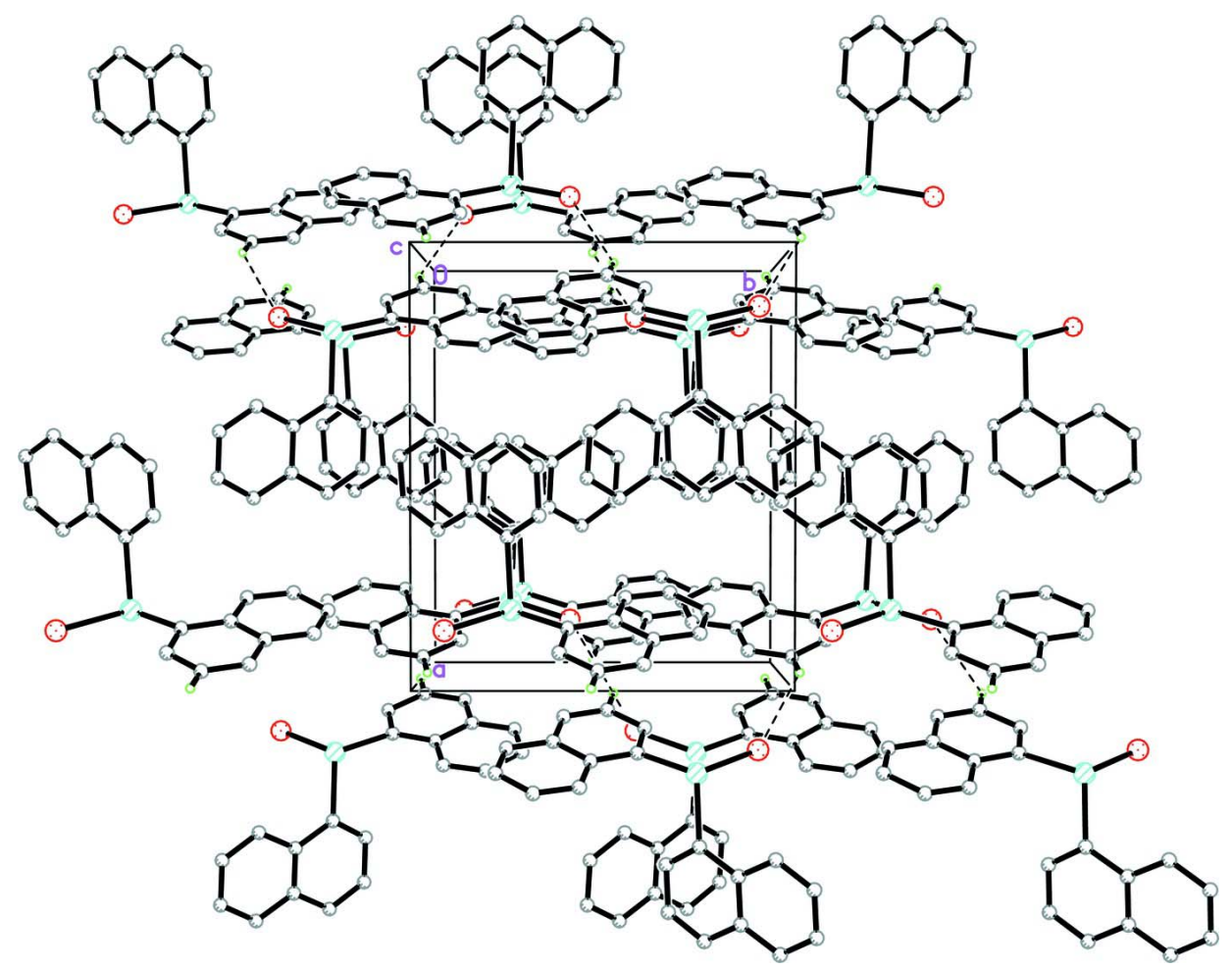

\section{Figure 2}

A crystal packing view of the title compound. Dashed lines indicate hydrogen bonds. $\mathrm{H}$ atoms not involved in the hydrogen bonds have been omitted for clarity.

\section{Bromidobis(naphthalen-1-yl)antimony(III)}

\section{Crystal data}

$\left[\mathrm{SbBr}\left(\mathrm{C}_{10} \mathrm{H}_{7}\right)_{2}\right]$

$M_{r}=455.97$

Monoclinic, $P 2_{1} / c$

Hall symbol: -P 2ybc

$a=12.7371(3) \AA$

$b=10.9189(3) \AA$

$c=11.6300$ (3) $\AA$

$\beta=92.661(1)^{\circ}$

$V=1615.70(7) \AA^{3}$

$Z=4$

\section{Data collection}

Bruker SMART APEXII CCD area-detector diffractometer

Radiation source: fine-focus sealed tube

Graphite monochromator

$\varphi$ and $\omega$ scans

Absorption correction: multi-scan

(SADABS; Bruker, 2009)

$T_{\min }=0.204, T_{\max }=0.597$
$F(000)=880$

$D_{\mathrm{x}}=1.875 \mathrm{Mg} \mathrm{m}^{-3}$

Mo $K \alpha$ radiation, $\lambda=0.71073 \AA$

Cell parameters from 9971 reflections

$\theta=2.5-32.2^{\circ}$

$\mu=4.17 \mathrm{~mm}^{-1}$

$T=100 \mathrm{~K}$

Block, yellow

$0.56 \times 0.33 \times 0.14 \mathrm{~mm}$

20528 measured reflections

4705 independent reflections

3936 reflections with $I>2 \sigma(I)$

$R_{\text {int }}=0.032$

$\theta_{\max }=30.0^{\circ}, \theta_{\min }=2.5^{\circ}$

$h=-17 \rightarrow 17$

$k=-15 \rightarrow 12$

$l=-16 \rightarrow 16$ 


\section{Refinement}

Refinement on $F^{2}$

Least-squares matrix: full

$R\left[F^{2}>2 \sigma\left(F^{2}\right)\right]=0.060$

$w R\left(F^{2}\right)=0.198$

$S=1.06$

4705 reflections

199 parameters

0 restraints

Primary atom site location: structure-invariant direct methods
Secondary atom site location: difference Fourier map

Hydrogen site location: inferred from neighbouring sites

$\mathrm{H}$-atom parameters constrained

$w=1 /\left[\sigma^{2}\left(F_{\mathrm{o}}^{2}\right)+(0.112 P)^{2}+12.2014 P\right]$ where $P=\left(F_{\mathrm{o}}{ }^{2}+2 F_{\mathrm{c}}{ }^{2}\right) / 3$

$(\Delta / \sigma)_{\max }<0.001$

$\Delta \rho_{\max }=3.68 \mathrm{e} \AA^{-3}$

$\Delta \rho_{\min }=-3.12$ e $\AA^{-3}$

Special details

Experimental. The crystal was placed in the cold stream of an Oxford Cryosystems Cobra open-flow nitrogen cryostat operating at 100.0 (1) K.

Geometry. All e.s.d.'s (except the e.s.d. in the dihedral angle between two l.s. planes) are estimated using the full covariance matrix. The cell e.s.d.'s are taken into account individually in the estimation of e.s.d.'s in distances, angles and torsion angles; correlations between e.s.d.'s in cell parameters are only used when they are defined by crystal symmetry. An approximate (isotropic) treatment of cell e.s.d.'s is used for estimating e.s.d.'s involving l.s. planes.

Refinement. Refinement of $F^{2}$ against ALL reflections. The weighted $R$-factor $w R$ and goodness of fit $S$ are based on $F^{2}$, conventional $R$-factors $R$ are based on $F$, with $F$ set to zero for negative $F^{2}$. The threshold expression of $F^{2}>\sigma\left(F^{2}\right)$ is used only for calculating $R$-factors(gt) etc. and is not relevant to the choice of reflections for refinement. $R$-factors based on $F^{2}$ are statistically about twice as large as those based on $F$, and $R$ - factors based on ALL data will be even larger.

Fractional atomic coordinates and isotropic or equivalent isotropic displacement parameters $\left(\AA^{2}\right)$

\begin{tabular}{lllll}
\hline & $x$ & $y$ & $z$ & $U_{\text {iso }} * U_{\text {eq }}$ \\
\hline Sb1 & $0.17759(3)$ & $0.74179(3)$ & $0.07848(3)$ & $0.02490(15)$ \\
Br1 & $0.13863(7)$ & $0.90878(7)$ & $-0.06577(8)$ & $0.0494(3)$ \\
C1 & $0.0943(5)$ & $0.6110(5)$ & $-0.1412(5)$ & $0.0257(11)$ \\
H1A & 0.0838 & 0.6928 & -0.1672 & $0.031^{*}$ \\
C2 & $0.0591(5)$ & $0.5128(6)$ & $-0.2125(5)$ & $0.0292(12)$ \\
H2A & 0.0267 & 0.5290 & -0.2862 & $0.035^{*}$ \\
C3 & $0.0717(5)$ & $0.3958(6)$ & $-0.1758(5)$ & $0.0261(11)$ \\
H3A & 0.0482 & 0.3304 & -0.2244 & $0.031^{*}$ \\
C4 & $0.1192(4)$ & $0.3695(5)$ & $-0.0659(5)$ & $0.0230(10)$ \\
C5 & $0.1307(5)$ & $0.2481(5)$ & $-0.0249(6)$ & $0.0288(13)$ \\
H5A & 0.1061 & 0.1822 & -0.0721 & $0.035^{*}$ \\
C6 & $0.1765(5)$ & $0.2234(6)$ & $0.0814(6)$ & $0.0313(13)$ \\
H6A & 0.1831 & 0.1413 & 0.1078 & $0.038^{*}$ \\
C7 & $0.2140(6)$ & $0.3218(6)$ & $0.1516(6)$ & $0.0326(13)$ \\
H7A & 0.2463 & 0.3050 & 0.2252 & $0.039^{*}$ \\
C8 & $0.2043(5)$ & $0.4399(6)$ & $0.1150(5)$ & $0.0289(12)$ \\
H8A & 0.2299 & 0.5044 & 0.1634 & $0.035^{*}$ \\
C9 & $0.1567(4)$ & $0.4681(5)$ & $0.0055(5)$ & $0.0212(10)$ \\
C10 & $0.1425(4)$ & $0.5910(5)$ & $-0.0364(5)$ & $0.0206(10)$ \\
C11 & $0.3440(4)$ & $0.7501(5)$ & $0.0575(5)$ & $0.0220(10)$ \\
C12 & $0.3925(5)$ & $0.6658(5)$ & $-0.0089(5)$ & $0.0244(10)$ \\
H12A & 0.3522 & 0.6024 & -0.0453 & $0.029^{*}$ \\
C13 & $0.5023(5)$ & $0.6718(6)$ & $-0.0241(5)$ & $0.0309(12)$ \\
& & & &
\end{tabular}




\begin{tabular}{lllll} 
H13A & 0.5354 & 0.6120 & -0.0695 & $0.037^{*}$ \\
C14 & $0.5603(5)$ & $0.7642(6)$ & $0.0268(6)$ & $0.0314(13)$ \\
H14A & 0.6339 & 0.7672 & 0.0171 & $0.038^{*}$ \\
C15 & $0.5130(5)$ & $0.8555(6)$ & $0.0936(5)$ & $0.0278(12)$ \\
C16 & $0.5719(5)$ & $0.9541(6)$ & $0.1423(6)$ & $0.0351(14)$ \\
H16A & 0.6452 & 0.9590 & 0.1310 & $0.042^{*}$ \\
C17 & $0.5250(6)$ & $1.0422(7)$ & $0.2053(6)$ & $0.0424(18)$ \\
H17A & 0.5653 & 1.1091 & 0.2354 & $0.051^{*}$ \\
C18 & $0.4171(6)$ & $1.0344(6)$ & $0.2257(6)$ & $0.0370(15)$ \\
H18A & 0.3853 & 1.0948 & 0.2715 & $0.044^{*}$ \\
C19 & $0.3575(5)$ & $0.9398(5)$ & $0.1799(5)$ & $0.0277(11)$ \\
H19A & 0.2847 & 0.9359 & 0.1940 & $0.033^{*}$ \\
C20 & $0.4029(4)$ & $0.8482(5)$ & $0.1120(5)$ & $0.0233(10)$ \\
\hline
\end{tabular}

Atomic displacement parameters $\left(\AA^{2}\right)$

\begin{tabular}{lllllll}
\hline & $U^{11}$ & $U^{22}$ & $U^{33}$ & $U^{12}$ & $U^{13}$ & $U^{23}$ \\
\hline Sb1 & $0.0235(2)$ & $0.0210(2)$ & $0.0304(2)$ & $-0.00274(13)$ & $0.00205(15)$ & $-0.00743(13)$ \\
Br1 & $0.0450(4)$ & $0.0284(4)$ & $0.0729(6)$ & $0.0000(3)$ & $-0.0185(4)$ & $0.0040(3)$ \\
C1 & $0.025(3)$ & $0.023(3)$ & $0.028(3)$ & $-0.006(2)$ & $-0.001(2)$ & $0.001(2)$ \\
C2 & $0.028(3)$ & $0.033(3)$ & $0.026(3)$ & $-0.005(2)$ & $-0.004(2)$ & $-0.004(2)$ \\
C3 & $0.027(3)$ & $0.027(3)$ & $0.025(2)$ & $-0.008(2)$ & $-0.001(2)$ & $-0.007(2)$ \\
C4 & $0.023(2)$ & $0.021(2)$ & $0.026(2)$ & $-0.0027(19)$ & $0.0031(19)$ & $-0.0039(19)$ \\
C5 & $0.032(3)$ & $0.015(2)$ & $0.039(3)$ & $-0.005(2)$ & $0.009(3)$ & $-0.004(2)$ \\
C6 & $0.031(3)$ & $0.021(3)$ & $0.042(4)$ & $0.000(2)$ & $0.006(3)$ & $0.005(2)$ \\
C7 & $0.041(3)$ & $0.028(3)$ & $0.028(3)$ & $0.001(3)$ & $-0.003(2)$ & $0.006(2)$ \\
C8 & $0.034(3)$ & $0.025(3)$ & $0.027(3)$ & $-0.004(2)$ & $-0.003(2)$ & $-0.003(2)$ \\
C9 & $0.020(2)$ & $0.019(2)$ & $0.025(2)$ & $-0.0038(18)$ & $0.0020(18)$ & $-0.0027(19)$ \\
C10 & $0.019(2)$ & $0.015(2)$ & $0.028(3)$ & $-0.0010(17)$ & $0.0013(18)$ & $-0.0054(18)$ \\
C11 & $0.018(2)$ & $0.022(3)$ & $0.026(2)$ & $-0.0024(18)$ & $-0.0040(19)$ & $0.0023(18)$ \\
C12 & $0.025(3)$ & $0.021(2)$ & $0.027(3)$ & $0.000(2)$ & $0.000(2)$ & $-0.0011(19)$ \\
C13 & $0.027(3)$ & $0.034(3)$ & $0.032(3)$ & $0.005(2)$ & $0.002(2)$ & $0.003(2)$ \\
C14 & $0.019(3)$ & $0.042(4)$ & $0.033(3)$ & $-0.001(2)$ & $0.000(2)$ & $0.007(2)$ \\
C15 & $0.027(3)$ & $0.028(3)$ & $0.027(3)$ & $-0.006(2)$ & $-0.009(2)$ & $0.010(2)$ \\
C16 & $0.033(3)$ & $0.035(3)$ & $0.036(3)$ & $-0.013(3)$ & $-0.012(2)$ & $0.010(3)$ \\
C17 & $0.056(4)$ & $0.031(3)$ & $0.037(3)$ & $-0.018(3)$ & $-0.026(3)$ & $0.011(3)$ \\
C18 & $0.053(4)$ & $0.024(3)$ & $0.033(3)$ & $-0.004(3)$ & $-0.017(3)$ & $-0.003(2)$ \\
C19 & $0.034(3)$ & $0.022(3)$ & $0.026(3)$ & $-0.001(2)$ & $-0.010(2)$ & $0.000(2)$ \\
C20 & $0.026(3)$ & $0.021(2)$ & $0.022(2)$ & $-0.004(2)$ & $-0.0084(19)$ & $0.0029(19)$ \\
& & & & & & \\
\hline & & & & & & \\
\hline
\end{tabular}

Geometric parameters $\left(\AA,{ }^{\circ}\right)$

\begin{tabular}{llll}
\hline $\mathrm{Sb} 1-\mathrm{C} 11$ & $2.146(6)$ & $\mathrm{C} 9-\mathrm{C} 10$ & $1.436(7)$ \\
$\mathrm{Sb} 1-\mathrm{C} 10$ & $2.155(5)$ & $\mathrm{C} 11-\mathrm{C} 12$ & $1.367(8)$ \\
$\mathrm{Sb} 1-\mathrm{B} 1$ & $2.5116(9)$ & $\mathrm{C} 11-\mathrm{C} 20$ & $1.438(7)$ \\
$\mathrm{C} 1-\mathrm{C} 10$ & $1.357(8)$ & $\mathrm{C} 12-\mathrm{C} 13$ & $1.420(9)$ \\
$\mathrm{C} 1-\mathrm{C} 2$ & $1.416(8)$ & $\mathrm{C} 12-\mathrm{H} 12 \mathrm{~A}$ & 0.9500 \\
$\mathrm{C} 1-\mathrm{H} 1 \mathrm{~A}$ & 0.9500 & $\mathrm{C} 13-\mathrm{C} 14$ & $1.369(10)$
\end{tabular}




\begin{tabular}{|c|c|c|c|}
\hline $\mathrm{C} 2-\mathrm{C} 3$ & $1.354(9)$ & $\mathrm{C} 13-\mathrm{H} 13 \mathrm{~A}$ & 0.9500 \\
\hline $\mathrm{C} 2-\mathrm{H} 2 \mathrm{~A}$ & 0.9500 & $\mathrm{C} 14-\mathrm{C} 15$ & $1.416(10)$ \\
\hline $\mathrm{C} 3-\mathrm{C} 4$ & $1.418(8)$ & $\mathrm{C} 14-\mathrm{H} 14 \mathrm{~A}$ & 0.9500 \\
\hline $\mathrm{C} 3-\mathrm{H} 3 \mathrm{~A}$ & 0.9500 & $\mathrm{C} 15-\mathrm{C} 16$ & $1.416(8)$ \\
\hline $\mathrm{C} 4-\mathrm{C} 5$ & $1.414(8)$ & $\mathrm{C} 15-\mathrm{C} 20$ & $1.431(8)$ \\
\hline $\mathrm{C} 4-\mathrm{C} 9$ & $1.429(7)$ & $\mathrm{C} 16-\mathrm{C} 17$ & $1.363(12)$ \\
\hline $\mathrm{C} 5-\mathrm{C} 6$ & $1.368(10)$ & $\mathrm{C} 16-\mathrm{H} 16 \mathrm{~A}$ & 0.9500 \\
\hline $\mathrm{C} 5-\mathrm{H} 5 \mathrm{~A}$ & 0.9500 & $\mathrm{C} 17-\mathrm{C} 18$ & $1.409(12)$ \\
\hline $\mathrm{C} 6-\mathrm{C} 7$ & $1.419(10)$ & C17-H17A & 0.9500 \\
\hline C6-H6A & 0.9500 & $\mathrm{C} 18-\mathrm{C} 19$ & $1.374(8)$ \\
\hline $\mathrm{C} 7-\mathrm{C} 8$ & $1.362(9)$ & $\mathrm{C} 18-\mathrm{H} 18 \mathrm{~A}$ & 0.9500 \\
\hline $\mathrm{C} 7-\mathrm{H} 7 \mathrm{~A}$ & 0.9500 & $\mathrm{C} 19-\mathrm{C} 20$ & $1.414(9)$ \\
\hline $\mathrm{C} 8-\mathrm{C} 9$ & $1.418(8)$ & $\mathrm{C} 19-\mathrm{H} 19 \mathrm{~A}$ & 0.9500 \\
\hline $\mathrm{C} 8-\mathrm{H} 8 \mathrm{~A}$ & 0.9500 & & \\
\hline $\mathrm{C} 11-\mathrm{Sb} 1-\mathrm{C} 10$ & $98.0(2)$ & $\mathrm{C} 9-\mathrm{C} 10-\mathrm{Sb} 1$ & $119.0(4)$ \\
\hline $\mathrm{C} 11-\mathrm{Sb} 1-\mathrm{Br} 1$ & $93.38(15)$ & $\mathrm{C} 12-\mathrm{C} 11-\mathrm{C} 20$ & $120.7(5)$ \\
\hline $\mathrm{C} 10-\mathrm{Sb} 1-\mathrm{Br} 1$ & $96.42(15)$ & $\mathrm{C} 12-\mathrm{C} 11-\mathrm{Sb} 1$ & $120.7(4)$ \\
\hline $\mathrm{C} 10-\mathrm{C} 1-\mathrm{C} 2$ & $121.4(5)$ & $\mathrm{C} 20-\mathrm{C} 11-\mathrm{Sb} 1$ & $118.6(4)$ \\
\hline $\mathrm{C} 10-\mathrm{C} 1-\mathrm{H} 1 \mathrm{~A}$ & 119.3 & $\mathrm{C} 11-\mathrm{C} 12-\mathrm{C} 13$ & $120.9(5)$ \\
\hline $\mathrm{C} 2-\mathrm{C} 1-\mathrm{H} 1 \mathrm{~A}$ & 119.3 & $\mathrm{C} 11-\mathrm{C} 12-\mathrm{H} 12 \mathrm{~A}$ & 119.5 \\
\hline $\mathrm{C} 3-\mathrm{C} 2-\mathrm{C} 1$ & $120.0(5)$ & $\mathrm{C} 13-\mathrm{C} 12-\mathrm{H} 12 \mathrm{~A}$ & 119.5 \\
\hline $\mathrm{C} 3-\mathrm{C} 2-\mathrm{H} 2 \mathrm{~A}$ & 120.0 & $\mathrm{C} 14-\mathrm{C} 13-\mathrm{C} 12$ & $119.6(6)$ \\
\hline $\mathrm{C} 1-\mathrm{C} 2-\mathrm{H} 2 \mathrm{~A}$ & 120.0 & $\mathrm{C} 14-\mathrm{C} 13-\mathrm{H} 13 \mathrm{~A}$ & 120.2 \\
\hline $\mathrm{C} 2-\mathrm{C} 3-\mathrm{C} 4$ & $121.0(5)$ & $\mathrm{C} 12-\mathrm{C} 13-\mathrm{H} 13 \mathrm{~A}$ & 120.2 \\
\hline $\mathrm{C} 2-\mathrm{C} 3-\mathrm{H} 3 \mathrm{~A}$ & 119.5 & $\mathrm{C} 13-\mathrm{C} 14-\mathrm{C} 15$ & $121.4(6)$ \\
\hline $\mathrm{C} 4-\mathrm{C} 3-\mathrm{H} 3 \mathrm{~A}$ & 119.5 & $\mathrm{C} 13-\mathrm{C} 14-\mathrm{H} 14 \mathrm{~A}$ & 119.3 \\
\hline $\mathrm{C} 5-\mathrm{C} 4-\mathrm{C} 3$ & $121.8(5)$ & $\mathrm{C} 15-\mathrm{C} 14-\mathrm{H} 14 \mathrm{~A}$ & 119.3 \\
\hline $\mathrm{C} 5-\mathrm{C} 4-\mathrm{C} 9$ & $118.9(5)$ & $\mathrm{C} 14-\mathrm{C} 15-\mathrm{C} 16$ & $121.6(6)$ \\
\hline $\mathrm{C} 3-\mathrm{C} 4-\mathrm{C} 9$ & $119.2(5)$ & $\mathrm{C} 14-\mathrm{C} 15-\mathrm{C} 20$ & $119.2(5)$ \\
\hline $\mathrm{C} 6-\mathrm{C} 5-\mathrm{C} 4$ & $121.5(6)$ & $\mathrm{C} 16-\mathrm{C} 15-\mathrm{C} 20$ & $119.2(6)$ \\
\hline $\mathrm{C} 6-\mathrm{C} 5-\mathrm{H} 5 \mathrm{~A}$ & 119.2 & $\mathrm{C} 17-\mathrm{C} 16-\mathrm{C} 15$ & $121.0(6)$ \\
\hline $\mathrm{C} 4-\mathrm{C} 5-\mathrm{H} 5 \mathrm{~A}$ & 119.2 & $\mathrm{C} 17-\mathrm{C} 16-\mathrm{H} 16 \mathrm{~A}$ & 119.5 \\
\hline $\mathrm{C} 5-\mathrm{C} 6-\mathrm{C} 7$ & $119.3(6)$ & $\mathrm{C} 15-\mathrm{C} 16-\mathrm{H} 16 \mathrm{~A}$ & 119.5 \\
\hline $\mathrm{C} 5-\mathrm{C} 6-\mathrm{H} 6 \mathrm{~A}$ & 120.4 & $\mathrm{C} 16-\mathrm{C} 17-\mathrm{C} 18$ & $120.2(6)$ \\
\hline $\mathrm{C} 7-\mathrm{C} 6-\mathrm{H} 6 \mathrm{~A}$ & 120.4 & $\mathrm{C} 16-\mathrm{C} 17-\mathrm{H} 17 \mathrm{~A}$ & 119.9 \\
\hline $\mathrm{C} 8-\mathrm{C} 7-\mathrm{C} 6$ & $120.9(6)$ & $\mathrm{C} 18-\mathrm{C} 17-\mathrm{H} 17 \mathrm{~A}$ & 119.9 \\
\hline $\mathrm{C} 8-\mathrm{C} 7-\mathrm{H} 7 \mathrm{~A}$ & 119.6 & $\mathrm{C} 19-\mathrm{C} 18-\mathrm{C} 17$ & $120.4(7)$ \\
\hline $\mathrm{C} 6-\mathrm{C} 7-\mathrm{H} 7 \mathrm{~A}$ & 119.6 & $\mathrm{C} 19-\mathrm{C} 18-\mathrm{H} 18 \mathrm{~A}$ & 119.8 \\
\hline $\mathrm{C} 7-\mathrm{C} 8-\mathrm{C} 9$ & $121.0(6)$ & $\mathrm{C} 17-\mathrm{C} 18-\mathrm{H} 18 \mathrm{~A}$ & 119.8 \\
\hline $\mathrm{C} 7-\mathrm{C} 8-\mathrm{H} 8 \mathrm{~A}$ & 119.5 & $\mathrm{C} 18-\mathrm{C} 19-\mathrm{C} 20$ & $121.0(6)$ \\
\hline $\mathrm{C} 9-\mathrm{C} 8-\mathrm{H} 8 \mathrm{~A}$ & 119.5 & $\mathrm{C} 18-\mathrm{C} 19-\mathrm{H} 19 \mathrm{~A}$ & 119.5 \\
\hline $\mathrm{C} 8-\mathrm{C} 9-\mathrm{C} 4$ & $118.4(5)$ & $\mathrm{C} 20-\mathrm{C} 19-\mathrm{H} 19 \mathrm{~A}$ & 119.5 \\
\hline $\mathrm{C} 8-\mathrm{C} 9-\mathrm{C} 10$ & $123.3(5)$ & $\mathrm{C} 19-\mathrm{C} 20-\mathrm{C} 15$ & $118.2(5)$ \\
\hline $\mathrm{C} 4-\mathrm{C} 9-\mathrm{C} 10$ & $118.3(5)$ & $\mathrm{C} 19-\mathrm{C} 20-\mathrm{C} 11$ & $123.7(5)$ \\
\hline $\mathrm{C} 1-\mathrm{C} 10-\mathrm{C} 9$ & $120.0(5)$ & $\mathrm{C} 15-\mathrm{C} 20-\mathrm{C} 11$ & $118.1(5)$ \\
\hline $\mathrm{C} 1-\mathrm{C} 10-\mathrm{Sb} 1$ & $120.4(4)$ & & \\
\hline
\end{tabular}




$\begin{array}{llll}\mathrm{C} 10-\mathrm{C} 1-\mathrm{C} 2-\mathrm{C} 3 & 1.2(9) & \mathrm{C} 10-\mathrm{Sb} 1-\mathrm{C} 11-\mathrm{C} 12 & -3.9(5) \\ \mathrm{C} 1-\mathrm{C} 2-\mathrm{C} 3-\mathrm{C} 4 & 0.4(9) & \mathrm{B} 1-\mathrm{Sb} 1-\mathrm{C} 11-\mathrm{C} 12 & -100.9(5) \\ \mathrm{C} 2-\mathrm{C} 3-\mathrm{C} 4-\mathrm{C} 5 & 178.4(6) & \mathrm{C} 10-\mathrm{S} 1-\mathrm{C} 11-\mathrm{C} 20 & 174.3(4) \\ \mathrm{C} 2-\mathrm{C} 3-\mathrm{C} 4-\mathrm{C} 9 & -1.6(9) & \mathrm{B} 1-\mathrm{S} 1-\mathrm{C} 11-\mathrm{C} 20 & 77.4(4) \\ \mathrm{C} 3-\mathrm{C} 4-\mathrm{C} 5-\mathrm{C} 6 & -179.9(6) & \mathrm{C} 20-\mathrm{C} 11-\mathrm{C} 12-\mathrm{C} 13 & 1.0(9) \\ \mathrm{C} 9-\mathrm{C} 4-\mathrm{C} 5-\mathrm{C} 6 & 0.2(9) & \mathrm{S} 1-\mathrm{C} 11-\mathrm{C} 12-\mathrm{C} 13 & 179.2(4) \\ \mathrm{C} 4-\mathrm{C} 5-\mathrm{C} 6-\mathrm{C} 7 & -0.5(10) & \mathrm{C} 11-\mathrm{C} 12-\mathrm{C} 13-\mathrm{C} 14 & -1.0(9) \\ \mathrm{C} 5-\mathrm{C} 6-\mathrm{C} 7-\mathrm{C} 8 & 0.4(10) & \mathrm{C} 12-\mathrm{C} 13-\mathrm{C} 14-\mathrm{C} 15 & -0.8(10) \\ \mathrm{C} 6-\mathrm{C} 7-\mathrm{C} 8-\mathrm{C} 9 & -0.1(10) & \mathrm{C} 13-\mathrm{C} 14-\mathrm{C} 15-\mathrm{C} 16 & -177.3(6) \\ \mathrm{C} 7-\mathrm{C} 8-\mathrm{C} 9-\mathrm{C} 4 & -0.2(9) & \mathrm{C} 13-\mathrm{C} 14-\mathrm{C} 15-\mathrm{C} 20 & 2.5(9) \\ \mathrm{C} 7-\mathrm{C} 8-\mathrm{C} 9-\mathrm{C} 10 & 178.7(6) & \mathrm{C} 14-\mathrm{C} 15-\mathrm{C} 16-\mathrm{C} 17 & 179.2(6) \\ \mathrm{C} 5-\mathrm{C} 4-\mathrm{C} 9-\mathrm{C} 8 & 0.2(8) & \mathrm{C} 20-\mathrm{C} 15-\mathrm{C} 16-\mathrm{C} 17 & -0.6(9) \\ \mathrm{C} 3-\mathrm{C} 4-\mathrm{C} 9-\mathrm{C} 8 & -179.8(5) & \mathrm{C} 15-\mathrm{C} 16-\mathrm{C} 17-\mathrm{C} 18 & 1.9(10) \\ \mathrm{C} 5-\mathrm{C} 4-\mathrm{C} 9-\mathrm{C} 10 & -178.7(5) & \mathrm{C} 16-\mathrm{C} 17-\mathrm{C} 18-\mathrm{C} 19 & -1.8(10) \\ \mathrm{C} 3-\mathrm{C} 4-\mathrm{C} 9-\mathrm{C} 10 & 1.3(8) & \mathrm{C} 17-\mathrm{C} 18-\mathrm{C} 19-\mathrm{C} 20 & 0.4(9) \\ \mathrm{C} 2-\mathrm{C} 1-\mathrm{C} 10-\mathrm{C} 9 & -1.5(9) & \mathrm{C} 18-\mathrm{C} 19-\mathrm{C} 20-\mathrm{C} 15 & 0.9(8) \\ \mathrm{C} 2-\mathrm{C} 1-\mathrm{C} 10-\mathrm{Sb} 1 & -172.3(4) & \mathrm{C} 18-\mathrm{C} 19-\mathrm{C} 20-\mathrm{C} 11 & -177.2(6) \\ \mathrm{C} 8-\mathrm{C} 9-\mathrm{C} 10-\mathrm{C} 1 & -178.6(6) & \mathrm{C} 14-\mathrm{C} 15-\mathrm{C} 20-\mathrm{C} 19 & 179.5(5) \\ \mathrm{C} 4-\mathrm{C} 9-\mathrm{C} 10-\mathrm{C} 1 & 0.2(8) & \mathrm{C} 16-\mathrm{C} 15-\mathrm{C} 20-\mathrm{C} 19 & -0.8(8) \\ \mathrm{C} 8-\mathrm{C} 9-\mathrm{C} 10-\mathrm{Sb} 1 & -7.6(7) & \mathrm{C} 14-\mathrm{C} 15-\mathrm{C} 20-\mathrm{C} 11 & -2.4(8) \\ \mathrm{C} 4-\mathrm{C} 9-\mathrm{C} 10-\mathrm{Sb} 1 & 171.2(4) & \mathrm{C} 16-\mathrm{C} 15-\mathrm{C} 20-\mathrm{C} 11 & 177.4(5) \\ \mathrm{C} 11-\mathrm{S} 1-\mathrm{C} 10-\mathrm{C} 1 & -105.6(5) & \mathrm{C} 12-\mathrm{C} 11-\mathrm{C} 20-\mathrm{C} 19 & 178.7(5) \\ \mathrm{Br} 1-\mathrm{Sb} 1-\mathrm{C} 10-\mathrm{C} 1 & -11.2(5) & \mathrm{Sb} 1-\mathrm{C} 11-\mathrm{C} 20-\mathrm{C} 19 & 0.5(7) \\ \mathrm{C} 11-\mathrm{Sb} 1-\mathrm{C} 10-\mathrm{C} 9 & 83.5(4) & \mathrm{C} 12-\mathrm{C} 11-\mathrm{C} 20-\mathrm{C} 15 & 0.7(8) \\ \mathrm{Br} 1-\mathrm{Sb} 1-\mathrm{C} 10-\mathrm{C} 9 & 177.8(4) & \mathrm{Sb} 1-\mathrm{C} 11-\mathrm{C} 20-\mathrm{C} 15 & -177.6(4) \\ & & & \end{array}$

Hydrogen-bond geometry $\left(\AA,{ }^{\circ}\right)$

\begin{tabular}{lllll}
\hline$D-\mathrm{H} \cdots A$ & $D-\mathrm{H}$ & $\mathrm{H} \cdots A$ & $D \cdots A$ & $D-\mathrm{H} \cdots A$ \\
\hline $\mathrm{C} 1-\mathrm{H} 1 A \cdots \mathrm{Br} 1$ & 0.95 & 2.71 & $3.408(6)$ & 130 \\
$\mathrm{C} 2-\mathrm{H} 2 A \cdots \mathrm{Br} 1^{\mathrm{i}}$ & 0.95 & 2.96 & $3.698(6)$ & 135 \\
\hline
\end{tabular}

Symmetry code: (i) $-x, y-1 / 2,-z-1 / 2$. 